\title{
Development of Dual-Actuated Stage for Positioning Applications
}

\author{
Prabha Niranjan $^{1}$, K. V. S. S. S. S. Sairam ${ }^{1}$, Shashikantha Karinka ${ }^{2}$ \\ ${ }^{1}$ Department of Electronics and Communication Engineering, \\ ${ }^{2}$ Department of Mechanical Engineering, \\ NMAMIT, Nitte. \\ prabhan@nitte.edu.in
}

\begin{abstract}
Piezoelectric actuators have been widely used in precision positioning applications because of their fast response, fine resolution, and large actuating forces. The displacement of piezoelectric actuators should be precisely controlled to use them in precision actuation stages. However to use these piezoelectric actuators in control systems, it is very much necessary to estimate the displacement of actuator based on their input voltage and also the inverse model. This paper presents Prandtl-Ishlinskii model for describing the nonlinearity of the actuator. Inverse Prandtl-Ishlinskii model will be used in the feed forward loop for the compensation of nonlinearity. This research work also includes development of a dual-actuated single stage to overcome the small travel range of piezoelectric stack actuators. The piezoelectric stack actuator is used for fine positioning and ball screw actuator is used for coarse positioning in the proposed dual-actuated stage. A closed loop control will be designed for the dual positioning stage, and this stage will be later applied for real-time wing flap control of aircraft model.
\end{abstract}

Keywords: Piezoelectric actuator; Prandtl-Ishlinskii model; Positioning; Closed loop control system; Displacement; Dual-Stage; Ball screw actuator.

\section{Introduction}

In recent years, there is lot of scope for research in ultra precision positioning applications. It includes diamond cutting, drilling machine, wing flap control in aircraft etc. Such ultra precision position systems require displacement control in the range of micrometers to millimeters.

In ultra-precision positioning, the piezoelectric actuators are used because of their good dynamic response and high resolution. It can be used for nano-positioning drives but it is bit tedious to provide higher load capacity. Whereas ball screw provides higher load capacity and stiffness but it fails in providing accuracy in sub-micrometer range [1],[2].

Hence for high precision machines, dual positioning systems have been developed where piezoelectric actuator is used for fine positioning and ball screw actuator is used for coarse positioning [3], [4], [5]. 
In this paper, the various hysteresis models are discussed in section 2 . The simulation model of piezoelectric actuator is presented in section 3 . The proposed dual-actuated single stage for positioning applications is given in section 4, followed by conclusion and future work.

\section{Hysteresis Modeling}

Piezoelectric actuators (PEAs) are widely used in variety of positioning applications due to their advantages of high positioning resolution in the range of micrometers to nanometers, large force, high dynamic response, high stiffness and compact design [6], [7], [8]. The major drawback of the PEA is its hysteresis nonlinearity that leads to positioning inaccuracy, and even instability of closed loop systems [9]. In order to increase the positioning accuracy of the PEA, there is a need to model the PEA, taking into consideration hysteresis and creep nonlinearities, which otherwise lead to large errors.

The various mathematical models which are popularly used to describe the hysteresis phenomenon like Preisach model, Prandtl-Ishlinskii (PI) model, Maxwell-slip model, etc. are proposed by various researchers [10].

In Preisach model, much of data is required to characterize the nonlinear systems that will influence the accuracy of the model. Obtaining inverse model is not easy in this type due to double integral present in the equation [11],[12],[13].

Maxwell-slip model operates with an elasto slide element comprising of mass less linear spring and mass less block those are susceptible to coulomb friction. One of the limitations with Maxwell-slip model is about hysteresis rising curve. The smart actuators need to start from a relaxed state that is not easily acquired in case of this model [14].

Many Researchers have used the sub class of Preisach model, known as PrandtlIshlinskii (PI) model for hysteresis compensation of PEA. The PI hysteresis model is the super position of several backlash operators, which are similar to the relay operators used in the Preisach model [15],[16]. The Preisach model is more accurate compared to PI model, but PI model is chosen due to availability of analytical inverse. In PI model only with few backlash operators, the hysteresis can be accurately modeled.

\section{Simulation Model}

PI hysteresis model is a phenomenological operator based model, used to describe the nonlinear relationship between the applied voltage and the resultant displacement of PEAs. In this model, the output is a weighted sum of many backlash operators. This model has been widely used for real time compensation of hysteresis nonlinearity in control applications due to its reduced complexity and the analytical form of inversion as compared to Preisach hysteresis model.

The backlash operator is the primary operator of the PI model, and it is defined in the discrete domain as follows:

$$
\mathrm{y}(\mathrm{k})=\mathrm{H}_{\mathrm{r}}\left[\mathrm{x}, \mathrm{y}_{0}\right](\mathrm{k})=\max \{\mathrm{x}(\mathrm{k})-\mathrm{r}, \min \{\mathrm{x}(\mathrm{k})+\mathrm{r}, \mathrm{y}(\mathrm{k}-1)\}\} .
$$


where, $\mathrm{x}=\mathrm{c} 1 * \mathrm{xdata}+\mathrm{c} 2$, where $\mathrm{c} 1$ and $\mathrm{c} 2$ are constants and $\mathrm{xdata}$ is the applied input to the model, $\mathrm{y}$ is the actuator response and $\mathrm{r}$ is the single threshold value of the backlash operator.

The initial condition of equation (1) is given by,

$$
y(0)=\max \left\{x(0)-r, \min \left\{x(0)+r, y_{0}\right\}\right\}
$$

The hysteresis loop can be modeled by a linearly weighted superposition of many backlash operators with different threshold and weight values, as given in equation (3).

$$
\mathrm{y}(\mathrm{k})=\mathrm{w}^{\mathrm{T}} \cdot \mathrm{H}_{\mathrm{r}}\left[\mathrm{r}, \mathrm{y}_{0}\right](\mathrm{k})
$$

where weight vector, $\mathrm{w}^{\mathrm{T}}=\left[\mathrm{w}_{1} \mathrm{w}_{2} \mathrm{w}_{3} \ldots . \mathrm{w}_{\mathrm{n}}\right]$ and initial value vector, $\mathrm{y}_{0}^{\mathrm{T}}=\left[\begin{array}{llll}\mathrm{y}_{01} & \mathrm{y}_{02} & \mathrm{y}_{03}\end{array}\right.$

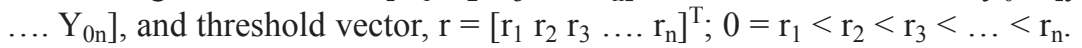

The threshold values of the backlash operators are selected within the applied voltage range, with equal intervals. The weight values decide the shape and size of the hysteresis loop, which are found by the experimental data. Also it is assumed that the actuator starts with its de-magnetized state. So the initial value is always zero.

\subsection{Parameter Estimation}

First the PEA response is measured by driving the actuator up to the full displacement range. In this study, $0-150 \mathrm{~V}$, input with $1 \mathrm{~Hz}$ sinusoidal signal is given to PEA and the corresponding displacement readings are noted. Then voltage and displacement readings are imported to MATLAB least-squares optimization program for parameter calculation with lsqcurvefit function.

This requires a user defined function to compute the vector valued function $\mathrm{F}(\mathrm{x}$, $x d a t a)$. This function will find coefficients $x$ that best fit the equation, $F(x, x d a t a)$. In this model, a total of 17 backlash operators are used in order to demonstrate the hysteresis loop. Geometric progression method is implemented, in order to choose the threshold parameters. The values of constants $\mathrm{c} 1$ and $\mathrm{c} 2$ are calculated based on equation $\mathrm{x}=\mathrm{c} 1$ * $\mathrm{xdata}+\mathrm{c} 2$, they are, $\mathrm{c} 1=0.973693604078441$ and $\mathrm{c} 2=-2.88447749468157$.

Threshold Value $\mathrm{r}_{\mathrm{i}}(\mathrm{V})$ and Weight Value $\left(\mathrm{w}_{\mathrm{i}}\right)$ are calculated based on equation (1) (3). The Inverse PI model can be obtained from the identified PI model. Because of the analytical inverse, the design of inverse PI model becomes more straightforward. The parameters $r_{i}$ and $w_{i}$, for the inverse PI model are determined based on equations (4)-(7).

$$
\begin{gathered}
\mathrm{H}^{-1}[\mathrm{y}(\mathrm{k})]=\mathrm{w}^{\mathrm{T}} \cdot \mathrm{H}_{\mathrm{r}}\left[\mathrm{y}, \mathrm{y}_{0}\right](\mathrm{k})=\sum_{\mathrm{i}=1}^{\mathrm{n}} \mathrm{w}_{\mathrm{i}}^{\prime} \cdot \max \left\{\mathrm{y}(\mathrm{k})-\mathrm{r}_{\mathrm{i}}^{\prime}, \min \left\{\mathrm{y}(\mathrm{k})+\mathrm{r}_{\mathrm{i}}^{\prime}, \mathrm{y}_{\mathrm{i}}^{\prime}(\mathrm{k}-1)\right\}\right\} . \\
\text { where, } \mathrm{r}_{\mathrm{i}}^{\prime}=\sum_{\mathrm{j}=1}^{\mathrm{i}} \mathrm{W}_{\mathrm{j}}\left(\mathrm{r}_{\mathrm{i}}-\mathrm{r}_{\mathrm{j}}\right), \mathrm{i}=1,2, \ldots \mathrm{n} . \\
\mathrm{y}_{\mathrm{oi}}^{\prime}=\sum_{\mathrm{j}=\mathrm{i}+1}^{\mathrm{i}} \mathrm{w}_{\mathrm{j}} \mathrm{y}_{0 \mathrm{i}}, \mathrm{i}=1,2, \ldots \mathrm{n}
\end{gathered}
$$


Prabha Niranjan, K. V. S. S. S. S. Sairam, Shashikantha Karinka

$$
\mathrm{w}_{1}^{\prime}=\frac{1}{\mathrm{w}_{1}}, \mathrm{w}^{\prime}=-\frac{\mathrm{w}_{\mathrm{i}}}{\left(\mathrm{w}_{\mathrm{i}}+\sum_{\mathrm{j}=2}^{\mathrm{i}} \mathrm{w}_{\mathrm{j}}\right)\left(\sum_{\mathrm{j}=1}^{\mathrm{i}-1} \mathrm{w}_{\mathrm{j}}\right)}, \mathrm{i}=2,3, \ldots \mathrm{n} .
$$

The values of constants are found based on equation, $\mathrm{x}=\mathrm{c} 1 * \mathrm{xdata}+\mathrm{c} 2$ they are, $\mathrm{c} 1=3.02098764835687$ and $\mathrm{c} 2=0.676064852409702$.

\subsection{Simulation Results}

The hysteresis nonlinearity of PEA is mathematically modeled using PI hysteresis model for sinusoidal signal of $150 \mathrm{~V}, 1 \mathrm{~Hz}$. The output of forward and inverse PI model for sinusoidal input signal is given in Figs. 1 and 2. Results show an error of $0.5 \%$ for forward model and $0.8 \%$ for inverse PI model, which is shown in Fig. 3. This shows that the PEA can be accurately modeled using sinusoidal input signal, due to fine variation at the peak and valley points of the signal.
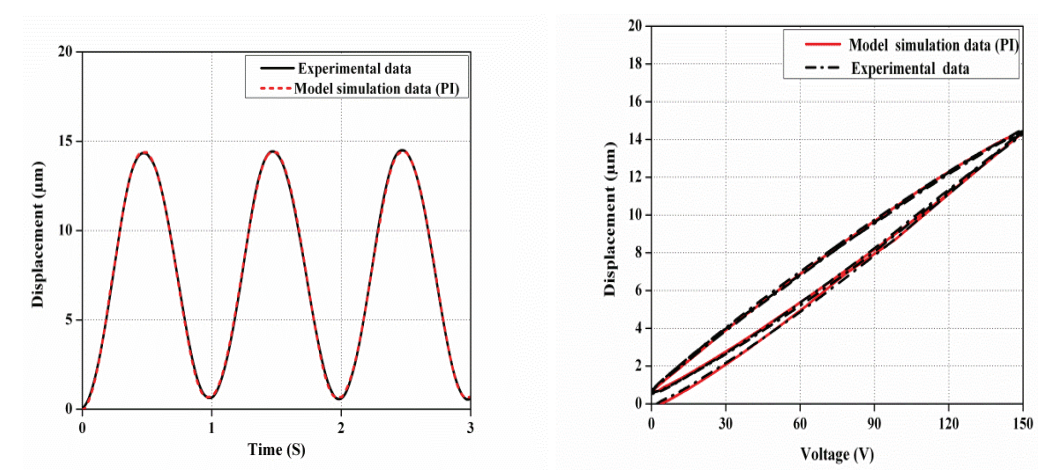

Fig. 1. a) PI hysteresis model output of PEA with $150 \mathrm{~V}, 1 \mathrm{~Hz}$ sinusoidal signal (b) Hysteresis loop.
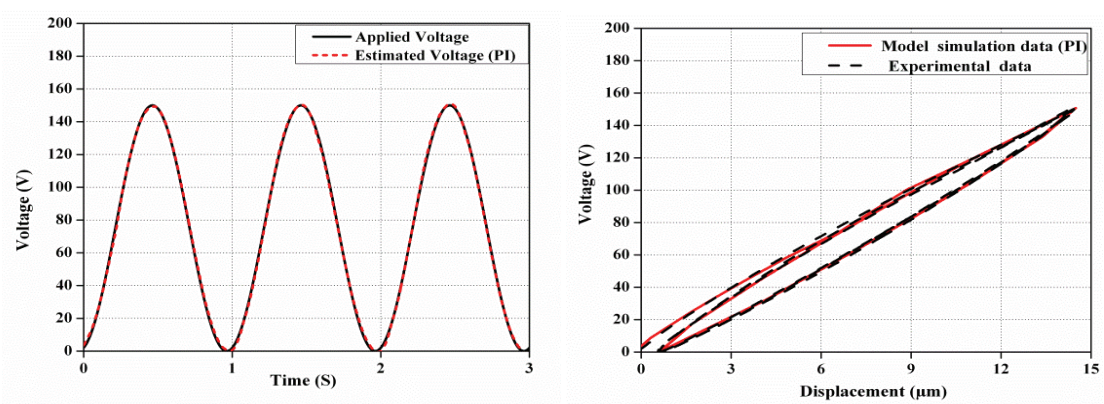

Fig. 2. a) PI inverse hysteresis model output of PEA with $150 \mathrm{~V}, 1 \mathrm{~Hz}$ sinusoidal signal (b) Inverse hysteresis loop. 

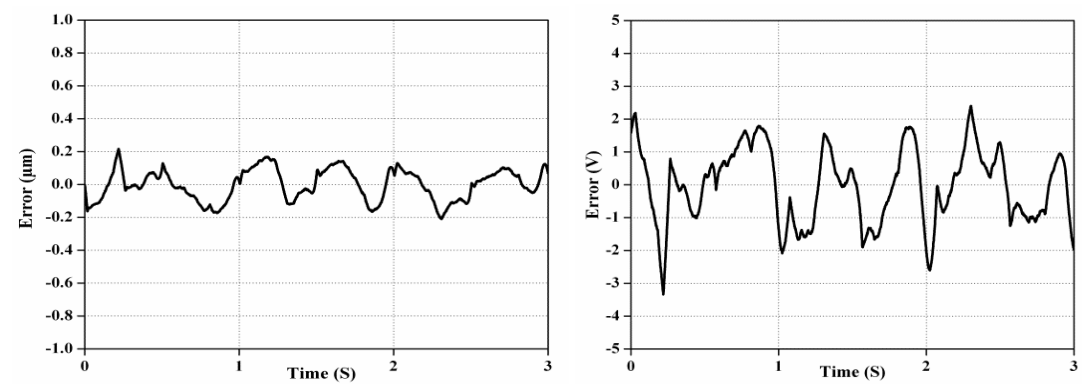

Fig. 3. Modeling error (a) PI forward model (b) PI inverse model.

\section{Proposed Method}

The block diagram of the experimental setup for the proposed work is as shown in Fig. 4. This setup has a piezoelectric stack actuator with very small travelling range within 20 micro meters, which can be used for fine positioning. And a ball screw with built in linear encoder is also used in the stage for coarse positioning. A single control system will be developed for the position control of the entire system. This system can be used for both micro and macro applications.

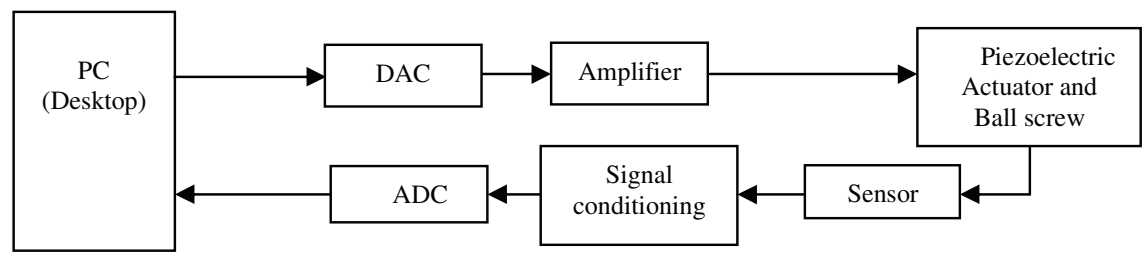

Fig. 4. Block diagram of proposed dual-actuated stage.

\section{Conclusion and Future Work}

The effectiveness of the hysteresis model is validated by comparing the experimental data with the simulation results. The modeling error is comparatively very small so that the simulated model can be further utilized in the feed forward loop for control design.

In the later part of the work, a dual-actuated stage will be developed with piezoelectric actuator for fine positioning and ball screw for coarse positioning. Control system will be developed for the real-time control of the dual stage. Developed scheme will be applied for the position control of wing flap of aircraft model as one of the example.

\section{References}

1. Matsubara, A., Fujitha,T., Kono, D., Tanaka, N., Watanabe, Y.: Nano-positioning drive with Piezoelectric Actuator Integrated into support bearing unit of Ball screw, pp. 323-326 (2007). 
2. Watanabe, S., Furutani, T., Yoshida, S.: Nanometer Positioning and its Micro-dynamics, Nanotechnology, Vol. 1, pp. 31-37 (2008).

3. Moriyama, S., Harada, T., Takanashi, A.: Precision X-Y Stage with a piezo-driven fine table, in the Bulletin of the Japan Society of Precision Engineering, Vol. 50, pp. 718-723 (1984).

4. Lee, C., Kim, S.: An Ultraprecision Stage for Alignment of Wafers in Advance Microlithography, in Precision Engineering, Vol. 21, pp. 335-345 (1997).

5. Pahk, H.J., Lee D.S., Park, J.H.,: Ulta Precision Positioning System for Servo Motor Piezo Actuator using DualnServo Loop and Digital Filter Implementation, in the International Journal of Machine Tools and Manufacture, Vol. 41, pp. 51-63 (2001).

6. Woronko, A., Huang, J.,: Piezoelectric Tool Actuator for Precision Machining on Conventional CNC Tuning Centers, Precision Engineering, Vol. 45, pp. 335-345 (2003).

7. Leang, K., and Devasia, S.: Design of Hysteresis Compensating Iterative Learning Control for Piezo Positioners: Application to Atomic Force Microscopes, Mechatronics, vol. 16, no. 4, pp. 141-158 (2006).

8. Gawthrop P., Bhikkaji B, and Mohimani S.: Physical Model Based Control of a Piezoelectric Tube for Nano Scale Positioning Applications, Mechatronics, vol. 20, no. 1, pp. 74-84 (2010).

9. Salapaka, S.: Scanning Probe Microscopy, IEEE Control Systems, vol. 28, no. 2, pp. 65-83 (2008).

10. Yang, M., G, Gu and Zhu, L.: High Bandwidth Tracking Control of Piezoactuated Nano Positioning Stages Using Closed Loop Input Shaper, Mechatronics, vol. 24, no. 6, pp. 724-733 (2014).

11. Ge, P., and Jouaneh, M.,: Tracking Control of a Piezoceramic Actuator, IEEE Trans. Control Syst. Technology, vol. 4, no. 3, pp. 209-216 (1996).

12. Mayergoyz, I. D and Friedman, G.,: Generalized Preisach Model of Hysteresis, IEEE Transactions on Magnetics, vol. 24, no. 1, pp. 212-217 (1998).

13. Song, G., Zhao, J., X. Zhou and J. A. De Abreu-Garcia: Tracking Control of a Piezoceramic Actuator with Hysteresis Compensation Using Inverse Preisach Model, IEEE/ASME Transactions on Mechatronics, vol. 10, no. 2, pp. 198-209 (2005).

14. Michel G. and Nikola C.: Modeling Piezoelectric Stack Actuators for Control of Micromanipulation, IEEE Control Systems, vol. 17, no. 3, pp. 69-79 (1997).

15. Bashash S., Jalili N.: Robust Multiple Frequency Trajectory Tracking Control of Piezoelectrically Driven Micro/Nano Positioning Systems, IEEE Transactions on Control Systems Technology, vol. 15, no. 5 (2007).

16. Kuhnen, K.: Modeling, Identification And Compensation Of Complex Hysteretic Nonlinearities: A Modified Prandtl-Ishlinskii Approach, European Journal of Control, vol. 9, no. 4, pp. 407-421 (2003). 